\title{
Effectiveness Bulletins
}

\section{Enhancing effective and acceptable purchaser and provider decisions: overview and methods}

\author{
A F Long, T A Sheldon
}

DHAs and FHSAs have been given the lead role in assessing the health needs of their residents, appraising service options, specifying a chosen pattern of service provision, placing contracts, and monitoring the provision of contracted services and their impact on the health of the population. To help this process the public health division of the Department of Health commissioned a series of bulletins on effectiveness to synthesise available evidence on chosen health care interventions. The project is based within the school of public health at the University of Leeds and is undertaken in collaboration with the Oncology Information Service (OIS) at the University of Leeds, the Centre for Health Economics (CHE) at the University of York, and the Research Unit of the Royal College of Physicians (RCP) in London.

\section{Overview of project}

The bulletins, which are published under the title Effective Health Care, are premised on the belief that scientific evidence of proved effectiveness and acceptability criteria should play an increasingly important part in decisions to purchase or provide particular services. The chosen topics will relate to areas where purchasers most need information and are likely to seek to encourage service changes. The first two target interventions - osteoporosis and rehabilitation after stroke - were selected by a steering group comprising purchasing managers; public health physicians; a FHSA general manager; and staff from $\mathrm{CHE}$, the RCP, and the research team. They were selected according to the following criteria: the intervention has a high resource implications; uncertainty or dispute exists over its effectiveness; the intervention probably has a large impact on health gain; and it has implications for both primary and secondary services. Ideas for future topics have been sought from recipients of the first bulletin.

Widely distributed to purchasers and providers and to professional, voluntary, and patient groups through direct mailing and the network of the RCP, the bulletins are, however, only one means of disseminating reviews on effectiveness and acceptability: workshops will aim at gaining commitment to the guidance provided by the bulletins, at helping in its translation to a local setting, and thus at facilitating the process of change.

\section{Methods: reviewing and synthesising the evidence}

The bulletins are produced by a stepwise process (box 1) based on established methodological checklists used within the Canadian Task Force on the Periodic Health Examination and the United States Preventive Services Task Force. ${ }^{1-3}$ Three principal criteria are used: clinical effectiveness, cost effectiveness, and acceptability.

STEP A: WHAT QUESTIONS SHOULD PUBLISHED STUDIES ANSWER?

Once a topic for a bulletin has been identified (for example, osteoporosis) and the purchasing question specified ("should population bone screening programmes for preventing fractures in postmenopausal women be established?") the next task is to clarify the areas and issues that must be addressed by available reports. This is done in three ways: firstly, an initial literature search is

$A$ What questions should published studies answer? Identify key studies and clinical experts Clarify causal pathways

$B$ How well do different types of study answer these questions?

Hierarchy of evidence (study types)

$C$ How valid is information from each study?

Internal validity

External validity

Statistical power

$D$ From review of the studies what does the evidence show?

Quantitative meta-analysis of clinical effectiveness

Evaluation of cost effectiveness

Issues of acceptability

$E$ What is overall impact of intervention?

Balance of risks and benefits

Health service context

Intersectoral implications

Target groups

Public health impact

Box 1 Review process 
conducted to access important studies; secondly, clinical experts are identified with help from the RCP; and, thirdly, the analytical tool of causal pathways is used to highlight the evidence that must be examined in order to evaluate the intervention. ${ }^{4}$

The literature search is undertaken in a structured manner. The research team and OIS staff explore the full extent of the subject, produce an agreed list of subject key words and search constraints, identify appropriate databases, and match the subject to database thesauri. Appropriate external databases and information centres are identified to conduct the initial search and to ensure that the retrieval of data is cross disciplinary, including clinical practice, health economics, health service management, and intersectoral implications, while recording the strategy used and the number of citations retrieved. The strategy is revised and updated in a systematic way.

This approach is supplemented by searches of "semi-published" or "grey" literature in the form of reports of the royal colleges, government departmental and health authority documents, items published outside the United Kingdom, or work in progress. OIS interrogates sources such as indexes and catalogues of official publications or the British Library System of Information in Grey Literature in Europe. External resources are also searched according to the original agreed strategy. The clinical experts identified help in locating published and unpublished material and in providing information on current and promising research in the topic area, as well as acting as an expert technical reviewer to the project.

STEP B: HOW WELL DO DIFFERENT TYPES OF STUDY ANSWER THESE QUESTIONS?

Within clinical epidemiology a hierarchy of types of studies has been delineated (box 2), ${ }^{1-35}$ which acknowledges that various study designs are subject to bias and other threats to internal validity, even in well designed studies.

\section{Rules of evidence}

I: Evidence obtained from at least one properly randomised controlled trial

II-1: Evidence obtained from well designed controlled trials without randomisation

II-2: Evidence obtained from well designed cohort or case-control analytical studies, preferably from more than one centre or research group

II-3: Evidence obtained from comparisons between times or places with or without the intervention. Dramatic results in uncontrolled experiments could be included in this section

III: Options of respected authorities, based on clinical experience, descriptive studies, or reports of expert committees

Source: Woolf et al 1990, table 3, p 898.

Box 2 Classifications of study designs, Canadian Task Force on the Periodic Health Examination, 1979
This hierarchy forms the basis for the critical appraisal of the literature within these bulletins, providing a way of clarifying how well the different types of study can shed light on the risks, benefits, and costs of the target intervention.

An intervention that works in a controlled study context such as that provided by a randomised controlled trial may not work in the standard conditions of routine practice. The distinction between efficacy and effectiveness is important. In particular, the effectiveness of an intervention may differ from its efficacy owing to factors related to the provider and the health care system (costs of care and resources in general) and to the patient (issues of acceptability and compliance with treatment).

\section{STEP C: HOW VALID IS INFORMATION FROM EACH STUDY?}

Each study requires systematic review, and well established methodological checklists ${ }^{5-8}$ are used to identify design strengths and flaws. Key issues addressed include internal validity ("do the results mean what they seem to?") and, in particular, the definition of variables and outcomes and their measurement and eventual data quality; the treatment of confounders and sample selection; statistical power; the clear specification of the hypothesis before inspection of the results; methods of data analysis; and external validity ("can I generalise?").

In this way the weight of evidence provided by each study is assessed. "Good" quality studies are extracted for examination against the mapping of the causal pathways and risks and benefits of the target intervention and in the light of the hierarchy of study types.

- Was a well defined question posed in answerable form?

- Was a comprehensive description of the competing alternatives given?

- Was there evidence that the programme's effectiveness had been established?

- Were all important and relevant costs and consequences for each alternative identified?

- Were costs and consequences measured accurately in appropriate physical units?

- Were costs and consequences valued credibly?

- Were costs and consequences adjusted for differential timing?

- Was an incremental analysis of costs and consequences of alternatives performed?

- Was a sensitivity analysis performed?

- Did the presentation and discussion of study results include all issues of concern to users?

Box 3 Abbreviated checklist for evaluating an economic appraisal, Drummond et al 1987 
STEP D: FROM REVIEW OF THE STUDIES, WHAT DOES THE EVIDENCE SHOW?

Analysis of the evidence is undertaken in three stages. Firstly, studies of "doubtful" validity are downgraded relative to "good" studies. A quantitative meta-analysis is undertaken when several comparable studies examine the efficacy or effectiveness of an intervention. As appropriate, the overall trend of the treatment effects for each individual study is presented in a table or graph, together with confidence intervals. The results from well performed meta-analyses will also be referenced and used.

Secondly, once the clinical effectiveness of the intervention has been established, the costs must be seriously investigated. The general approach outlined by Drummond et $a l^{9}$ is used to evaluate published economic appraisals (box 3). In many instances, however, an economic appraisal may be lacking. Attempts will be made to provide insight into cost implications and indicate specifically the need for further research. Attention will focus not only on the economic consequences to the health services but also to the community, other sectors, and the patient.

Thirdly, the potential acceptability of the target intervention is assessed. From a clinical perspective this relates to the notions of compliance and uptake ("will patients comply with the treatment regimen or attend the screening programme?") More broadly, take up of a service reflects consumer perceptions of the acceptability of the intervention. Attitudes and perceptions about the clinical condition and side effects of the intervention will affect the demand for and utilisation of services. Though many of the studies of consumer acceptability are probably in the form of surveys or ethnographic accounts of care or of illness, or both (and thus may not be widely perceived in the medical arena as presenting "hard" evidence) they must be taken into account in providing health care services. If compliance for an effective intervention is expected to be low ways of enhancing its acceptability must be addressed.

STEP E: WHAT IS THE OVERAIL IMPACT OF THE INTERVENTION?

The final stage in the review and synthesis process is to make an informed judgement of the overall effectiveness and acceptability of the intervention and its likely impact and to identify gaps in knowledge. Firstly, the balance of risks and benefits must be assessed: "will the intervention do more good than harm?" Possible adverse effects of the intervention are considered and thus potential increases in the incidence of non-target interventions. ${ }^{2}$ Secondly, the health service context of clinical practice is examined to translate the clinical and cost effectiveness recommendations into purchasing policy. Social acceptability and managerial and intersectoral implications are clarified. Potential and appropriate target groups for the intervention are identified, as far as the evidence allows. Thirdly, the individual and public health impact of the intervention is addressed. Establishing the effectiveness of a procedure does not automatically mean that the overall health of the population is (appreciably) improved. Highly effective interventions applied to small high risk groups may have less impact on the nation's health than a more modestly effective intervention for a target condition in which there is a greater burden of suffering.

\section{Conclusion}

The key task of the project is to clarify the effectiveness and acceptability of the target interventions and to produce recommendations based on interpretation of the current evidence according to agreed and explicit criteria. The aim is to provide purchasers and providers with current and scientifically defensible information. Sound purchasing and clinical practice will require the interpretation of these guidelines in the light of an analysis of the health needs of the local population and social and political factors. As Jenicek observed, "Even if political decisions finally override recommendations, well organised homework is necessary."10

We thank the following members of the project and research teams for their comments: Dr R Cartwright, Professor $\mathrm{H}$ Cuckle, Dr A Dowell, Professor M Drummond, Professor D Hunter, Dr S Ibbotson, and Dr C Pollock.

1 Canadian Task Force on the Periodic Health Examination The periodic health examination. Can Med Assoc 1979;121:1139-254.

2 Woolf SH, Battista RN, Anderson GM, Logan AG, Wang E, and Canadian Task Force on the Periodic Health Examination. Assessing the clinical effectiveness of preventive maneuvers: analytic principles and systematic methods in reviewing evidence and developing clinical practice recommendations. I Clin Epidemiol 1990;43:

3 US Preventive Services Task Force. Guide to clinical preventive services: an assessment of the effectiveness of 169 interventions. Boston: Williams and Wilkins, 1989

4 Battista RN, Fletcher SW. Making recommendations on preventive practices: methodological issues. In: Battista $\mathrm{RN}$, Lawrence RS, eds. Implementing preventive services. New York: Oxford University Press, 1988;53-67.

5 Sackett DL, Haynes RB, Tugwell P. Clinical epidemiology: Boston: Little, Brown, 198

6 Woolf RN. Interim manual for clinical practice guidelinc development. Agency for Health Care Policy and

7 Chalmers TC, Smith H Jr, Blackburn B, Silverman B, Schroeder B, Reitman D, et al. A method for assessing the quality of a randomised controlled trial. Controlled the quality of a random

8 Lichtenstein MJ, Mulrow CD, Elwood PC. Guidelines for reading case-control studies. Foumal of Chronic Diseases 1987; 40:893-903.

9 Drummond MF, Stoddart GL, Torrance GW. Methods for economic evaluation of health care programmes. Oxford: Oxford University Press, 1987.

10 Jenicek J. Meta-analysis in medicine. Where we are and where we want to go. 7 Clin Epidemiol 1989;42:35-44. 